\title{
Le bivalve invasif asiatique Corbicula fluminea (Heterodonta, Sphaeriacea, Corbiculidae) dans le bassin hydrographique de la Seine (France) : première prospection systématique et hypothèse sur la colonisation
}

\section{The invasive Asian bivalve Corbicula fluminea (Heterodonta, Sphaeriacea, Corbiculidae) in the drainage basin of the Seine (France) : first systematic prospecting and hypothesis on the colonization}

\author{
Thierry Vincent ${ }^{(1)}$ et Virginie Brancotte ${ }^{(2)}$ \\ (1) Muséum d'histoire naturelle, place du Vieux-Marché, 76600 LE HAVRE. \\ mel : thierry.vincent@ville-lehavre.fr \\ (2) Les Millériaux, 45360 PIERREFITTE-ĖS-BOIS. \\ mel : vbrancotte@aol.com
}

Résumé. - Au cours de l'été 2000 , le mollusque dulçaquicole Corbicula fluminea a été trouvé dans plusieurs sites le long de la Seine, dans trois de ses affluents et dans cinq de ses canaux. En Seine, l'espèce se répartit de l'amont vers l'aval entre Bray-sur-Seine et Poses. Le genre Corbicula n'a pas encore été observé dans la zone estuarienne. Les données de terrain sont présentées dans cette note. L'expansion est discutée et la répartition analysée.

Mots-clés. - Mollusque, dulçaquicole, fleuve, affluents, canaux.

\begin{abstract}
During summer 2000 , the freshwater clam Corbicula fluminea was found at several locks in the Seine river (France), in three of its tributaries and five of the connecting canals. In the Seine, the species is found from downstream of Bray-sur-Seine to upstream of Poses. Corbicula has not yet invaded the estuary. The data are documented, the colonization is discussed and the distribution is analysed.
\end{abstract}

Key-words. - Mollusc, freshwater, river, tributaries, connecting canals. 


\section{INTRODUCTION}

Le genre Corbicula regroupe des mollusques bivalves hétérodontes dulçaquicoles et d'eau saumâtre, eurythermes largement répartis en Afrique, en Asie et en Australie. Ellis (1978, p. 38) indique que l'espèce $C$. fluminalis est éteinte en Europe, bien que présente dans différents gisements fossilifères de France, du Danemark, de l'Allemagne, etc. II précise qu'une espèce proche, $C$. fluminea, a été introduite aux USA en provenance de l'est de l'Asie. Signalé dès 1938 en Amérique du Nord comme espèce allochtone, il n'a cessé, depuis cette date, de coloniser les fleuves de différents états. Deux espèces auraient été récemment identifiées - Corbicula fluminea (Müller, 1774 ) et Corbicula fluminalis (Müller, 1774) - dans le réseau hydrographique européen (Kinzelbach, 1991), en particulier au Portugal (Mouthon, 1981; Nagel, 1989), en Espagne (Araujo et al., 1993), en Allemagne (Jungbluth, 1996; Nagel, 1997), en Hollande (Bij de Vaate \& GreijdanusKlaas, 1990), en Belgique (Swinnen et al., 1998) et au Luxembourg (Dhur \& Massard, 1995) ${ }^{\prime \prime}$.
Corbicula fluminea a été détecté en France en 1977 (Mouthon, in litt, fide Chevallier, 2000) ou au début des années 80 (Mouthon, 1981; Cémagref, 1982). Morton (1987, p. 105) a confirmé que les individus observés en France dans la Dordogne par Mouthon appartenaient à l'espèce $C$. fluminea. Le caractère invasif de cette espèce permettait de penser qu'elle allait rapidement coloniser les principaux fleuves et canaux de I'hexagone. Cette colonisation peut se résumer ainsi :

1977 : cours inférieur de la Dordogne (Mouthon, fide Chevallier, 2000)

1980: Dordogne (Mouthon, 1981; Cémagref, 1982) ${ }^{(2 i}$

1985: Dronne et Garonne (Cémagref, 1986 in Fontan \& Meny, 1995)

1989 : canal du Midi (Girardi, 1990) Charente (Jourde, fide Chevallier, 2000)

1990: estuaire de la Loire (Baudet, fide Gruet, 1992)

Vézère (Fontan \& Meny, 1995) '31

haut Rhône (Cémagref, 1990 in Khalanski, 1997)

1991: Gers (Fontan \& Meny, 1995) Adour (Fontan \& Meny, 1995)

(1) Nagel (1989, p. 17) pose l'hypothese de Corbicula fluminalis, au Portugal, comme espèce allochtone et invasive ou comme espèce relicte.

(2) Nous (T.V.) confirmons la présence de l'espèce $C$. fluminea jusqu'à l'amont de Souillac. en rive droite à Raysse de Passat (bras mort) et La Borie en rive gauche (Lot) (collecte du 24 août 2000).

(3) Nous (T.V.) confirmons la présence de l'espèce $C$. fluminea sur la Vézere jusqu'en amont du Bugue (Malmussou) (collecte du 14 juillet 2000). Une recherche jusqu'a Saint-Pantaléon-del'Arche et Brive-la-Gaillarde est restée négative. L'implantation n'a pas été observée : peut-être à cause d'une eau trop polluée (Cémagref, 1982). 
1993: canal latéral à la Garonne (Dubois, 1995b)

1994 : Lot (Fontan \& Meny, 1995) (4) Moselle (juillet 1994 fide Bachmann et al., 1997 ; Khalanski, 1997) (5)

Meuse (Swinnen et al., 1998)

1995: canal Rhin-Rhône-nord de Mulhouse (Bauer, fin mai 1995, fide Nagel, 1997)

1996 : Aude et Tarn (Bertrand, fide Chevallier, 2000)

Rhin (début février 1996.; Nagel, 1997)

1997 : Seine à Paris (Bertrand, fide Chevallier, 2000)

basse Loire (Carré \& Berger, 2000) ${ }^{(6)}$

Les informations sur l'expansion de Corbicula fluminea dans le réseau hydrographique français se font plus discrètes depuis trois ou quatre ans, bien que l'espèce ait probablement continué à se répandre.

En ce qui concerne la Seine, seule la donnée de Bertrand datant de 1997 (quai d'Orléans, Paris), rapportée par Chevallier (2000) est connue.

La Seine, augmentée de six principaux affluents, draine la majeure partie du bassin parisien dont la superficie est de $78650 \mathrm{~km}^{2}$ environ. Le bassin hydrographique de la Seine est important. II nous a donc paru intéressant d'engager une recherche de terrain systématique sur le réseau hydrographique séquanien afin d'affiner les connaissances sur la répartition de Corbicula fluminea dans la Seine et ses principaux affluents. Au total, 121 stations ont ainsi été prospectées (fig. 1).

\section{MATÉRIEL ET MÉTHODE}

La Seine a été prospectée depuis l'embouchure jusqu'aux environs de Troyes. Les principaux affluents en rive gauche (Eure, Loing, Yonne) et en rive droite (Oise, Marne, Aube) ont également fait l'objet d'une investigation.

Tous les substrats ont été inspectés (pierres, graviers, sables et vase) associés ou non à des macrophytes. Les zones de concentration de coquilles, sur les plages, les îlots, les radiers et dans les ventres des méandres ont été particulièrement parcourus. Ce sont les espèces visibles à l'œil nu lors de ramassages à pied ou à l'aide du troubleau qui ont été conservées et identifiées.

(4) Nous (T.V.) confirmons la présence de l'espèce $C$. fluminea à Cahors (collecte du 16 juillet 2000).

(5) Nous (T.V.) confirmons la présence de l'espèce $C$. fluminea dans la Moselle, à la hauteur de l'usine électrique de La Maxe (près de Metz). Nous ajoutons que nous avons également trouvé en sympatrie $C$. fluminalis, les deux espèces étant présentes en très forte densité (collecte du 24 septembre 2000).

(6) Nous (V.B.) confirmons la présence de l'espèce C. fluminea en Loire moyenne jusqu'à Châtillon-sur-Loire (collecte du 23 août 2000). 


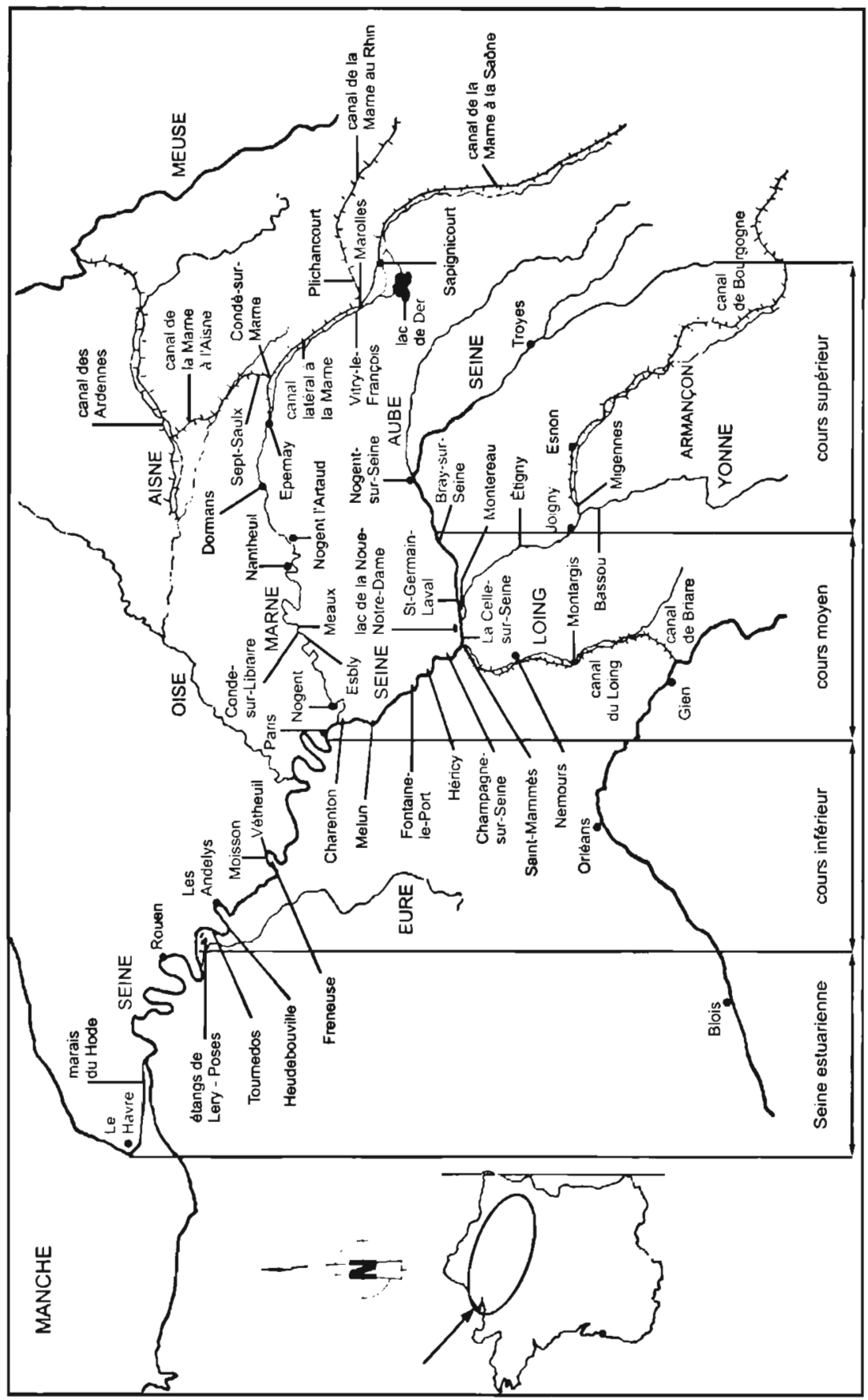

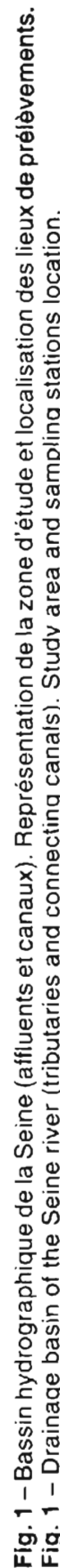


Lorsque les peuplements de Corbicula le permettaient, un décompte à été tenté afin d'obtenir une densité. L'espèce pouvant s'enfouir profondément - environ $20 \mathrm{~cm}$ - dans le sédiment meuble (Dubois, 1995a, p. 14) le substrat n'a cependant pas été fouillé en profondeur. Seules les rives de la Seine et des affluents ont été prospectées. Le fond des chenaux, tant de la Seine que des affluents, qui sont souvent des lieux très fréquentés par la batellerie, n'a pas été contrôlé. En revanche, le fond des canaux, la plupart au gabarit Freyssinet (canal de la Marne à L'Aisne ; canal de I'Yonne à la Saône; canal latéral du Loing, etc.), a été prospecté à l'aide d'un troubleau à long manche (6m).

\section{RÉSULTAT}

Les stations sur la Seine sont regroupées par secteurs; chaque affluent est distingué.

\subsection{La Seine}

La Seine a un cours de 776 kilomètres. Nous avons procédé au découpage du fleuve en différents tronçons :

a - Seine estuarienne : du Havre jusqu'au barrage de Poses. L'estuaire stricto sensu, près du Havre, et en rive droite, est occupé par le Marais du Hode;

b-cours inférieur : du barrage de Poses jusqu'à l'aval de Paris; c - cours moyen : depuis l'amont de Paris jusqu'au barrage de Jaulnes;

d - cours supérieur: du barrage de Jaulnes jusqu'à Troyes et au-delà.

\section{a - Seine estuarienne}

17 stations ont été prospectées depuis les bassins portuaires et les canaux de l'arrière-port du Havre, y compris la rivière la Lézarde et le canal de Tancarville, jusqu'au barrage de Poses, en amont de Rouen. Un coin d'eau salée s'insinue dans l'estuaire, jusqu'à la hauteur de Tancarville ou de Villequier selon le coefficient des marées et le débit de la Seine. Les eaux saumâtres pénètrent donc de $30 \mathrm{~km}$ environ dans l'espace estuarien. La marée dynamique est, quant à elle, sensible jusqu'audelà de Rouen mais se trouve arrêtée par le barrage de Poses.

Le genre Corbicula n'a été détecté sur aucune des stations prospectées, ni dans l'arrière-port du Havre, ni en basse Seine. C'est pourtant dans un environnement sensiblement identique que les premiers spécimens ont été collectés au Portugal dans les années 80 par $C$. Reis, dans l'estuaire du Tage (Mouthon, 1981). Les premiers spécimens français, provenant de la basse Dordogne, ont été trouvés en eau douce (Mouthon, 1981; Cémagref, 1982). C'est également dans la zone de marée dynamique du haut estuaire de la Loire que Baudet, en 1990, puis Stéphant, en 1991, enfin Gruet, en 1992, ont collecté les premières valves de Corbicula (Gruet, 1992). 


\section{b - Cours inférieur de la Seine}

Ce secteur s'étend du barrage de Poses à l'aval de Paris (le Vésinet). 24 stations ont été prospectées le 18 juillet 2000. Cinq d'entre elles ont permis de collecter les premiers spécimens de Corbicula fluminea de la Seine-aval.

Sur l'ensemble de ces 5 stations, les coquilles sont petites (en moyenne, $L: 12 \mathrm{~mm} ; \mathrm{h}: 10 \mathrm{~mm}$ ) - les individus sont donc jeunes - et la population peu nombreuse. La dispersion des individus ne nous a pas permis de procéder à une approche de densité. Une prospection des étangs de Léry (près de Poses) qui sont d'anciennes gravières transformées en bases aquatiques de loisir (par exemple le lac des deux Amants; lac du Mesnil) n'a pas permis d'observer Corbicula fluminea. Si le site est colonisé, il pourra se révéler de première importance pour l'expansion de l'espèce comme c'est le cas de la base de loisir de la Grande-Paroisse, en aval de Montereau (voir ci-après). II reste à noter toutefois que le lac des deux Amants (400 ha) n'est pas en communication avec la Seine, contrairement au lac du Mesnil ( $35 \mathrm{ha}$ ) qui est relié à un drain, lui-même en relation avec un canal de décharge à la Seine. C'est par ailleurs sur ce même tronçon de fleuve que se situe une unité de production thermique d'électricité (Porcheville). II était intéressant de savoir si l'espèce était déjà présente en nombre à cet endroit. Corbicula fluminea a colonisé des circuits des centrales thermiques en France, par exemple Cattenom et
La Maxe, sur la Moselle, Golfech sur la Garonne et Bugey sur le Rhône (Khalanski, 1997, p. 398-399; et comm. pers, février 2001).

\section{c - Cours moyen de la Seine}

Ce secteur s'étend de l'amont de Paris, jusqu'au barrage de Jaulnes, en amont de Bray-sur-Seine. 14 stations ont été prospectées les 8,9 et 10 août 2000. Corbicula est extrêmement présent à Corbeil-Essonnes, Melun ou Montereau.

Les densités observées à Melun varient de 80 à $100 \mathrm{ind} . / \mathrm{m}^{2}$ sur plusieurs centaines de mètres. La prédation de Corbicula - et dans une moindre mesure d'Unio pictorum par les rats musqués Ondatra zibethica (Linné, 1766) est le plus significatif le long des berges empierrées juste en amont de Melun. Des milliers de coquilles vides sont abandonnés au pied des aires d'alimentation des rongeurs.

À la confluence du Loing et de la Seine, à Saint-Mammès, les Corbicula fluminea sont nombreux mais en moindre densité qu'en aval (50 à $\left.80 \mathrm{ind} / \mathrm{m}^{2}\right)$. Au niveau de la base aquatique (51 ha.) de la Noue-NotreDame (commune de la Grande-Paroisse), le peuplement, très jeune ( $L x$ $\mathrm{h}: 11 \times 10 \mathrm{~mm}$ ), atteint des densités de 60 à 80 ind. $/ \mathrm{m}^{2}$. Cette base est en relation avec la Seine par différents canaux d'alimentation et de décharge. À la confluence de la Seine avec l'Yonne à Montereau, l'espèce est également très présente, sans qu'il nous ait été possible de chiffrer la densité moyenne. 
À partir de l'amont de Montereau, le peuplement de Corbicula en Seine est de l'ordre de 1 à 20 ind./m². L'espèce tend donc à diminuer en densité. Quelques spécimens ont cependant encore été collectés sur les bancs de sable et le long des berges à la hauteur de Bray-sur-Seine.

\section{d - Cours supérieur de la Seine}

Le barrage de Jaulnes semble créer une rupture brutale dans le pouvoir invasif de Corbicula fluminea. L'espèce n'est plus présente dans nos collectes, à partir du barrage et plus en amont jusqu'à Troyes.

\subsection{Les affluents de la Seine}

\subsubsection{L'Eure}

Cet affluent de la rive gauche, long de $225 \mathrm{~km}$, trouve sa confluence à l'aval de Caudebec-lès-Elbeuf. Les prospections à la recherche de Corbicula fluminea sont restées négatives.

\subsubsection{L'Oise}

Cet affluent de la rive droite, long de $302 \mathrm{~km}$, trouve sa confluence à Conflans-Sainte-Honorine. Les prospections à la recherche de Corbicula fluminea sont restées négatives. Cela peut paraître surprenant car l'Aisne, affluent de l'Oise, est reliée à la Meuse par le canal des Ardennes. $C$. fluminea a été trouvé dans le canal de la Marne à l'Aisne lors de notre prospection du 24 août 2000 (T.V.).

\subsubsection{La Marne}

Cet affluent de la rive droite, long de $525 \mathrm{~km}$, trouve sa confluence à Charenton-le-Pont. II est doublé par le canal latéral à la Marne qui rejoint la Marne à Épernay. Dans ce canal latéral se jette le canal de la Marne à l'Aisne. A la hauteur de Vitry-le-François, le canal latéral à la Marne se subdivise en canal de la Marne au Rhin et canal de la Marne à la Saône. 37 stations ont été parcourues les 24 et 25 août 2000. Corbicula fluminea n'a été collecté dans la Marne que de Charenton-le-Pont à Épernay. En amont de cette ville l'espèce ne semble pas présente. En revanche elle est bien représentée dans le canal latéral à la Marne, avec des densités pouvant atteindre $50 \mathrm{ind} . / \mathrm{m}^{2}$. Corbicula a également été trouvé dans le canal de l'Aisne à la Marne (Condé-sur-Marne), et au moins jusqu'à Sept-Saulx (voir ci-dessus : Oise). L'espèce a également été trouvée en nombre (35 à 40 ind. $/ \mathrm{m}^{2}$ ) dans le canal de la Marne à la Saône, au moins jusqu'à Sapignicourt. Malgré notre recherche de Marolles à Plichancourt, l'espèce n'a pu être collectée dans le canal de la Marne au Rhin. Nous rappelons que l'espèce est toutefois présente dans le Rhin depuis 1996, au moins, de même que dans le canal du Rhin au Rhône depuis 1995 (Nagel, 1997).

\subsubsection{Le Loing}

Cet affluent de la rive gauche, long de $166 \mathrm{~km}$ trouve sa confluence à 
Saint-Mammès. II est doublé par le canal du Loing et le canal de Briare.

Une recherche de Corbicula, tant sur le Loing ( 9 stations) que sur le canal de Briare puis le canal du Loing (7 stations), s'est avérée négative. Nous rappelons que l'espèce est cependant bien présente à la confluence du Loing avec la Seine à Saint-Mammès. De même, l'espèce a été trouvée par l'un de nous (V.B.) à Briare, dans le canal.

\subsubsection{L'Yonne}

Cet affluent de la rive droite, long de $295 \mathrm{~km}$, trouve sa confluence à Montereau. Corbicula fluminea est présent tout au long de son cours, depuis Montereau jusqu'aux abords d'Auxerre, au moins (Bassou). La découverte d'une coquille de $L 32 \times h$ $30 \mathrm{~mm}$ (Joigny; V.B., 9 août 2000) laisse à penser que la colonisation est ancienne et que le milieu est très favorable au développement de l'espèce. L'Yonne est reliée au canal de Bourgogne à Migennes et met en contact la Seine avec le réseau hydrographique du Rhône par la Saône. Des Corbicula fluminea ont été trouvés à l'entrée du canal de Bourgogne, par l'un de nous (T.V.) le 10 août 2000. L'espèce est d'ailleurs présente dans le canal au moins jusqu'à Esnon.

\subsubsection{L'Aube}

Cet affluent de la rive droite, long de $248 \mathrm{~km}$, trouve sa confluence à Pont-sur-Seine. Aucun Corbicula flu- minea n'y a été observé, ni à la confluence, ni plus haut sur son cours.

\section{DISCUSSION}

Le parcours systématique de la Seine, des affluents et des canaux entre les mois de juillet et de septembre 2000 permet de définir la répartition de Corbicula fluminea dans le réseau hydrographique séquanien.

La distribution apparaît irrégulière et limitée à une partie du fleuve et de ses affluents. Pour expliquer sa distribution restreinte, la première hypothèse voudrait que la limitation soit due à un manque de plasticité écologique de l'espèce. La seconde hypothèse serait que la colonisation de la Seine aval par Corbicula fluminea n'en est qu'à son début.

\section{1 Étude de la distribution}

Corbicula fluminea se répartit en amont et en aval de Paris, depuis le barrage de Jaulnes sur le cours moyen, jusqu'au barrage de Poses sur le cours inférieur. Le barrage de Jauines marque donc - pour le moment - la limite amont de présence de l'espèce. Fontan et Meny (1995, p. 37) ont insisté sur la difficulté que semble rencontrer ce bivalve à franchir les barrages. Ils indiquent que "pour passer ces obstacles, l'intervention de l'homme paraît néces saire". La colonisation du cours supérieur de la Seine pourrait donc 
n'être qu'une question de temps, sachant que le fleuve est doté à cet endroit d'une écluse et de canaux permettant le franchissement du barrage à la batellerie commerciale et de tourisme.

En aval du cours, le barrage de Poses marque également la limite de répartition de l'espèce. Le genre Corbicula étant euryhalin, la colonisation de la basse Seine apparaît là encore simplement soumise au temps.

Pour Chevallier (2000) Corbicula fluminea est ubiquiste, peu soucieux du potentiel hydrogène et assez eurytherme. Bachmann et al. (1997, p. 381) ont cependant démontré pour la Moselle que la granulométrie du substrat est nettement sélective, les sites colonisés sont plutôt ceux à substrats fins. Dubois (1995a) et Fontan et Meny (1995), ont montré que Corbicula fluminea pourrait être limité par certains paramètres physico-chimiques: température; faible ou très forte teneur en calcium; présence d'ammoniaque, etc. ${ }^{(7)}$. L'absence de pic thermique dans des rivières dont les eaux sont fraîches et courantes semble être une cause de non colonisation (et de non reproduction) ainsi que Fontan et Meny (op. cit.) ont pu le constater dans le bassin de la Garonne. Il pourrait en être de même dans le réseau hydrographique séquanien, en particulier dans l'Eure et le Loing.
Nous pensons que le débit des affluents auquel est liée la force du courant est un facteur qui pourrait également limiter l'expansion de l'espèce vers l'amont voir s'opposer à la colonisation de certains cours d'eau. En effet si les contre-courants de rives peuvent permettre à quelques larves de ce bivalve de remonter le cours sur une distance de quelques mètres à quelques dizaines de mètres, le débit doit cependant entraîner la plupart des larves vers l'aval et s'opposer à la progression naturelle de l'espèce vers l'amont.

\subsection{Distribution et rôle des affluents}

La distribution montre que l'espèce, outre sa limitation actuelle par les barrages, est répartie entre I'Yonne, en rive gauche, et la Marne, en rive droite. Les différentes stations réparties le long de l'Yonne permettent de juger de l'état de colonisation ancienne de cet affluent. II en est de même pour le canal latéral à la Marne, largement colonisé et pour la Marne, depuis Épernay, vers l'aval, jusqu'à sa confluence avec la Seine.

Nous pensons qu'il faut voir dans l'Yonne, plus exactement dans le canal de Bourgogne, et dans la Marne, plus précisément, dans le canal latéral à la Marne, un rôle de vecteur et de nurserie qui a permis à Corbicula

(7) Morton (1987) a démontré, pour C. fluminea étudié à Hongkong que différents écomorphes pouvaient être liés aux paramètres physico-chimiques de l'eau, en particulier le $\mathrm{pH}$ et la température. 
fluminea de commencer sa conquête d'un nouveau bassin hydrographique.

Nous développons dans un autre article le rôle qu'ont pu jouer les canaux dans la colonisation du bassin séquanien (Brancotte \& Vincent, 2001, à paraître).

\subsection{Remarques concernant la morphologie des coquilles collectées en Seine}

Morton (1987), pour les Corbicula fluminea de Hongkong, et Chevallier (2000, p. 17), pour ceux de France, insistent sur le polymorphisme de l'espèce; un rapport entre la longueur $(L)$ et la hauteur (h) permet cependant de caractériser Corbicula fluminea si $L>h$, ce qui se vérifie chez tous les spécimens que nous avons collectés aussi bien en Seine que dans les différents affluents et canaux du bassin hydrographique séquanien. V. Bachmann, dans sa thèse soutenue en juin 2000, a mis en évidence, à l'aide d'une formule mathematique, un coefficient de discrimination entre les deux espèces ( $C$. fluminea et $C$. fluminalis) en Moselle basé sur la hauteur $h$, la longueur $L$ et l'épaisseur $E$ en $\mathrm{mm}$. Cette étude implique le prélèvement d'un nombre élevé de coquilles pour que les mensurations soient statistiquement fiables (Bachmann, comm. pers., septembre 2000). En ce qui nous concerne cela n'a pu être réalisé, la colonisation par $C$. fluminea était en- core très récente en Seine en aval de Paris.

Une bonne description des Corbicula de la Dordogne et du Tage a été donnée par Mouthon (1981). En ce qui concerne la coloration externe des valves, Mouthon (1981) puis Girardi (1990) indiquent une couleur variable, généralement vert olive à brun-noir. Nous avons observé, en aval de Paris, des petits individus à périostracum foncé, mais à umbo érodé et donc blanchâtre. En amont de Paris, en Seine, dans les affluents et les canaux, les individus, sont plutôt clairs, à dominante olivâtre, avec parfois des ombrages brunâtres en bouffées de pipe sur les valves.

Nous avons constaté sur les spécimens collectés à Melun, le 8 août 2000 , des aberrations morphologiques. Les déformations affectent les valves droites et gauches. Une étude concernant cette particularité sera publiée prochainement (Vincent \& Brancotte, à paraître).

\section{CONCLUSION}

La malacocénose séquanienne s'est donc bien enrichie d'une nouvelle espèce - Corbicula fluminea dont l'expansion est encore en cours. $\mathrm{Ce}$ bivalve allochtone pourrait, à terme, entrer en concurrence avec la malacofaune autochtone. Un cas identique d'invasion avait déjà été enregistré au XIX" siècle avec Dreissena polymorpha (Pallas, 1771). 
Il est raisonnable de penser que la colonisation du bassin hydrographique de la Seine par Corbicula fluminea est due à une arrivée de ce mollusque bivalve par l'intermédiaire des canaux - canal latéral à la Marne et canal de Bourgogne - qui ont mis en contact certains affluents de la Seine - l'Yonne et la Marne - avec des sites colonisés appartenant à d'autres bassins hydrographiques, en particulier le bassin du Rhin, et peut-être celui du Rhône.

Si la colonisation de l'aval du fleuve (cours inférieur et Seine estuarienne) est en cours, la découverte par l'un de nous (T.V.) de petits spécimens entre Rouen et Paris, nous le confirme, en revanche l'expansion vers l'amont et en particulier le saut du barrage de Jaulnes nécessitera peut-être un peu plus de temps; la distribution de Corbicula sur l'ensemble du réseau hydrographique de la Seine semble inéluctable.

\section{REMERCIEMENTS}

Nous tenons à remercier la Ville du Havre qui nous a facilité les missions de recherche sur le terrain; nous remercions également $M$. M. Khalanski, EDF Recherche \& Développement à Chatou; $M$. le Docteur K.-O. Nagel; M. V. Bachmann, Démoécologie, CREUM, Université de Metz; M. J. Mouthon, Cémagref, Groupement de Lyon, pour ses précieux renseignements; M. C. Charrier, cellule environnement du CETMEF, Ministère de l'équipement; M. H. Chevallier, Centre d'Écologie à Fusternouau; M. J.-A. Massard, pour son aide lors de nos recherches bibliographiques; $M$. le directeur du
Cémagref, Groupement de Bordeaux et Mme Ch. Gardes, Service documentation; M. M. Dao, Cémagref, groupement d'Anthony, service documentation; M. H. Girardi, malacologue.

\section{RÉFÉRENCES BIBLIOGRAPHIQUES}

Araujo (R.), Moreno (D.) \& Ramos (M.A.) 1993 - The Asiatic clam Corbicula fluminea (Müller, 1774) (Bivalvia, Corbiculidae) in Europe. Am. Malac. Bull., $10(1): 39-49$.

Bachmann (V.), Usseglio-Polatera (P.), Cegielka (E.), Wagner (P.), Poinsaint (J.-F.) \& Moreteau (J.-C.) 1997 - Premières observations sur la coexistence de Dreissena polymorpha, Corophium curvispinum et Corbicula spp. dans la rivière Moselle. Bull. Fr. Pêch. Piscic., 344-345 : 373-384.

Bij de Vaate (A.) \& Greijdanus-Klaas (M.) 1990 - The Asiatic Clam Corbicula fluminea (Müller, 1774) (Pelecipoda, Corbiculidae) a new immigrant in the Netherlands. Bull. zoöl. Mus. Univ. Amsterdam, 12 (12) : 173-178.

Brancotte (V.) \& Vincent (T.) - Rôle des canaux de navigation dans l'invasion du réseau hydrographique français par Corbicula sp. Bulletin français de la pêche et de la protection des milieux aquatiques - à paraître.

Carré (F.) \& Berger (A.) 2000 - Quelques nouvelles malacologiques des pays de la Loire moyenne (région Centre, France). Vertigo, 7 (1997) : 35-43.

CÉMAGREF 1982 - Étude hydrobiologique de la Dordogne. Ministère de l'Agriculture / Ministère de l'Environnement. Cémagref Section qualité des eaux, groupement de Bordeaux (éd.) : $265 \mathrm{pp}, 72 \mathrm{pp}$ d'annexes.

Chevallier (H.) 2000 - Taxonomie des Corbicula (Bivalvia, Corbiculidae) in- 
troduites dans le sud-ouest de la France. Vertigo, 7 (1997) : 15-21.

Dhur (G.) \& Massard (J.-A.) 1995 - Étude historique et faunistique des invertébrés immigrés ou introduits dans la Moselle luxembourgeoise et ses affluents. Bull. Soc. Nat. Luxemb., 96 : 127-156.

Dubois (Ch.) 1995a - Corbicula fluminea: un mollusque opportuniste. Adour-Garonne, 63: 13-16.

Dubois (Ch.) 1995b - Biologie et démoécologie d'une espèce invasive, Corbicula fluminea (Mollusca, Bivalvia) originaire d'Asie: étude in situ (canal latéral à la Garonne, France) et en canal expérimental. Thèse de l'université Paul Sabatier, Toulouse : 169 pp.

Ellis (A.E.) 1978 - British freshwater Bivalve Mollusca. Keys and notes for the identification of the species. Linnean Society of London. British Fauna, n' 11, Academic Press (ed.), London: $109 \mathrm{pp}$.

Fontan (B.) \& Meny (J.) 1995 - Note sur l'invasion de Corbicula fluminea dans le réseau hydrographique de la région Aquitaine et précisions sur son spectre écologique. Vertigo, $5: 31-44$.

Girardi (H.) 1990 - Deux bivalves d'eau douce récents pour la faune française (Mollusca, Bivalvia). Bull. Soc. Ét. Sc. Nat. Vaucluse, 1989-1990: 87-93.

Gruet (Y.) 1992 - Un nouveau mollusque bivalve pour notre région: Corbicula sp. (Heterodonta, Sphaeriacea) Bull. Soc. Sc. Nat. ouest de la France, $\mathrm{N}^{\text {elin }}$ série, 14 (2) : 37-43.

Jungbluth (J.H.) 1996 - Einwanderer in der Molluskenfauna von Deutchland. In Gebhardt, Kinzelbach \& Shmidt-Fischer (Hrsg.). - Gebietsfremde Tierarten. Auswirkungen auf einheimische Arten, Lebensgemeinschaften und
Biotope. Situationanalyse. Ecomed (ed.) : $314 \mathrm{pp}$.

Khalanski (M.) 1997 - Conséquences industrielles et écologiques de l'introduction de nouvelles espèces dans les hydrosystèmes continentaux: la moule zébrée et autres espèces invasives. Bull. Fr. Pêche Piscic., 344345 : 385-404.

Kinzelbach (R.) 1991 - Die Körbchenmuscheln Corbicula fluminalis, Corbicula fluminea und Corbicula fluviatilis in Europa (Bivalvia, Corbiculidae). Mainzer Naturw. Archiv, 29 : 215-228.

Morton (B.) 1987 - Polymorphism in Corbicula fluminea (Bivalvia, Corbiculoidea) from Hong-Kong. Malacological Review, $20: 105-127$.

Mouthon (J.) 1981 - Sur la présence en France et au Portugal de Corbicula (Bivalvia, Corbiculidae) originaire d'Asie. Basteria, 45 : 109-116.

Mouthon (J.) 1994 - Fréquences et densités des espèces de mollusques dans les cours d'eau français. Vertigo, 4 : 19-28.

Mouthon (J.) 1997 - Les mollusques dulcicoles du bassin de la Loire, premier inventaire et caractéristiques des peuplements du fleuve. Vertigo, $5: 3-12$.

Nagel (K.-O. von) 1989 - Ein weiterer Fundort von Corbicula fluminalis (Müller, 1774) (Mollusca : Bivalvia) in Portugal. Mitt. disch. malakozool. Ges., 44-45: 17.

Nagel (K.-O. von) 1997 - Corbicula - Notizen. Mitt. dtsch. malakozool. Gest., $59: 11-13$.

Swinnen (F.), Leynen (M.), Sablon (R.), Duvivier (L.) \& Vanmaele (R.) 1998 The Asiatic clam Corbicula (Bivalvia, Corbiculidae) in Belgium. Bull. de I'Institut Royal des Sc. Nat. de Belgique, Biologie, 68 : 47-53. 